\title{
Poloxamer 407 based-nanoparticles for controlled release of methotrexate
}

\author{
Sofia Moura ${ }^{1}$, Jennifer Noro ${ }^{1}$, Patrícia Cerqueira, Carla Silva, Artur Cavaco-Paulo, Ana Loureiro* \\ Centre of Biological Engineering, University of Minho, Campus de Gualtar, 4710-057 Braga, Portugal
}

\section{A R T I C L E I N F O}

\section{Keywords:}

Poloxamer 407-based nanoparticles

Methotrexate di-ethylated

Methotrexate-Poloxamer 407 conjugate

Drug release

Cancer therapy

\begin{abstract}
A B S T R A C T
Poloxamer 407 (P407)-based nanoparticles were produced by the high pressure homogenization method for the encapsulation and delivery of methotrexate (MTX), aiming intravenous therapeutic applications. The surface of these nanoparticles was functionalized by conjugation of P407 with folic acid (FA) or with MTX, which served as targeting ligand agents. MTX-P407 conjugate was also developed to increase the final drug cargo. Two hydrophobic derivatives of MTX, MTX di-ethylated ester (MTX-OEt) and the ionic complex MTX-dimethyldioctadecylammonium bromide (MTX-DODAB) were produced and entrapped onto P407-based nanoparticles. All formulations developed revealed a monodisperse character comprising small and narrow nanoparticles ( $<100 \mathrm{~nm}$ ). P407 nanoparticles (functionalized with FA) and MTX-P407 nanoparticles, both loaded with MTXOEt, demonstrated a slow drug release profile. The effect of lipase from Aspergillus oryzae on the hydrolysis of the linkage between the P407 and MTX, and consequent MTX release profile, was also evaluated. We observed a controlled and slow release of MTX ( $<50 \%$ of release after 11 days) in the presence of enzyme. These MTX-P407 nanoparticles loaded with MTX-OEt induced a great effect against Caco- 2 cancer cells $(\approx 40 \%$ of cell death after $72 \mathrm{~h}$ of incubation), demonstrating higher efficiency than the free MTX at the same concentration.
\end{abstract}

\section{Introduction}

Nanomedicine has gained interest as a mean to potentiate the advantages of efficient drug delivery (Huang et al., 2018), decreasing the risk of toxicity to the normal tissues and organs of the patient (Alam et al., 2015). The clever use of nanoparticles has revolutionized how drugs are formulated and delivered (Rizvi and Saleh, 2018). Nanoparticles have been synthesized using various different methods depending of its application and type of drugs to be encapsulated (Pinto Reis et al., 2006). Loureiro and co-workers, reported a novel method based on high pressure homogenization of a biphasic system (aqueous phase and vegetable oil) to produce stable P407-based nanoparticles of small size (Loureiro et al., 2018). Poloxamers are poly(ethylene oxide) (PEO)-poly(propylene oxide) (PPO)-poly(ethylene oxide) type of block copolymers generally expressed as $\mathrm{PEO}_{\mathrm{x}}-\mathrm{PPO}_{\mathrm{y}}-\mathrm{PEO}_{\mathrm{x}}$ (Kabanov et al., 2002b), where $x$ and $y$ designate the total average number of PEO and PPO repeat units, respectively (Kedar et al., 2010). This arrangement results in amphiphilic copolymers (Kabanov et al., 2002a). Reviews that address Poloxamers have explored specific structures, such as hydrogels and Poloxamer-modified particles (Bodratti and Alexandridis, 2018). Due to their lateral PEG hydrophilic chains, Poloxamers provide steric stabilization and prevention of protein adsorption, resulting in less phagocytized nanoparticles and longer half-life in the systemic circulation (Alexis et al., 2008). Another exceptional feature is that Poloxamers inhibit efflux action of P-glycoprotein (Chen et al., 2013), which is associated to multidrug resistance (MDR) (Kedar et al., 2010).

MTX is one of the most studied and effective chemotherapeutic agents available to treat many tumours and autoimmune diseases (Abolmaali et al., 2014). MTX has also been described as a dual-acting molecule, due to its high structural similarity to FA, and it can be used as targeting agent (Loureiro et al., 2016). However, limitations as toxicity and drug resistance associated to adverse side-effects, poor aqueous solubility and short circulation half-life of the free acid form of MTX have been limiting its therapeutic applications (Choi et al., 2018; Gulfam et al., 2017). To overcome these drawbacks and improve the drug efficacy and pharmacokinetics many studies have been carried out. One of the strategies is the development of prodrugs by chemical modification of the MTX. For example, MTX dodecyl ester derivative was synthetized by esterification of the $\alpha$ - and $\gamma$-carbonyl groups of the glutamic acid aiming to increase its lipophilic behaviour, for a higher encapsulation in a lipid formulation (Moura et al., 2011). Xie and coworkers synthetized MTX-PEG-DSPE to produce micellar nanoparticles. This MTX prodrug was used as dual-acting agent, acting simultaneously as tumour targeting ligand and as anticancer agent, revealing to be an

\footnotetext{
* Corresponding author.

E-mail address: aloureiro@ceb.uminho.pt (A. Loureiro).

${ }^{1}$ Both authors contributed equally.
} 
interesting example of a drug delivery system (Xie et al., 2018). The amphiphilic properties of Poloxamer have also been explored on its conjugation with MTX for the production of polymeric micelles, allowing a sustained release of MTX (Ren et al., 2015).

In this work, P407-based nanoparticles were developed in order to optimize the encapsulation of MTX and its controlled delivery (Loureiro et al., 2018). Two hydrophobic MTX derivatives were developed, MTXOEt and an ionic complex of MTX-DODAB, and encapsulated on P407based nanoparticles. These hydrophobic derivatives revealed higher encapsulation onto P407-based nanoparticles ability compared to the commercial MTX. The conjugation of P407 with MTX was also assessed to produce nanoparticles containing MTX as a dual-acting agent and to increase the concentration of MTX on the final formulation. The biological effect of these nanoparticles against cancer cells (Caco-2) was evaluated.

\section{Materials and methods}

FA, P407, 4-dimethylaminopyridine (DMAP), pyridine, lipase from Aspergillus oryzae ( $>100000 \mathrm{U} / \mathrm{g}$ ), dialysis tubes (cut-off $1 \mathrm{kDa}$ ), 2,5dihydroxybenzoic acid (DHB) (suitable for matrix assisted laser desorption ionization time-of-flight mass spectroscopy (MALDI-TOF MS)), $\mathrm{NaOH}$ and all culture media and supplements were purchased from Sigma-Aldrich, USA. $N, N^{\prime}$-dicyclohexylcarbodiimide (DCC), DODAB and MTX were obtained from TCI Chemicals, Belgium. Dialysis membranes (cut-off $2 \mathrm{kDa}$ ), acetonitrile (high performance liquid chromatography (HPLC) grade) and $\mathrm{HCl} 37 \%$ were obtained from Fisher Scientific, USA. Anhydrous dimethyl sulfoxide (DMSO) dried under molecular sieves, was bought from Acros Organics, Belgium. Phosphate buffered saline (PBS) was acquired from Biochrom GmbH, Germany. Ultrapure water was obtained from a Milli-Q Water Purification System, Germany. Human colonic epithelial cells (Caco-2 cell line) (ATCC, HTB37) were obtained from American Type Culture Collection (ATCC), UK. T75 flasks and 96-well tissue culture polystyrene plates were acquired from SPL Life Sciences, Korea. CellTiter $96^{\circledR}$ AQueous Non-Radioactive Cell Proliferation [3-(4,5-dimethylthiazol-2-yl)-5-(3-carboxymethoxyphenyl)-2-(4-sulfophenyl)-2H-tetrazolium, inner salt] (MTS) assay was purchased from Promega, USA.

\subsection{Synthesis procedures}

\subsubsection{Synthesis of MTX-P407 and FA-P407 conjugates}

The synthesis of FA-P407 and MTX-P407 conjugates was performed following the procedure previously described by Loureiro et al. (Loureiro et al., 2018). Briefly, commercial FA or MTX (0.459 g, $1.01 \mathrm{mmol}$ ) was dissolved in anhydrous DMSO $(20 \mathrm{~mL})$ with DCC (0.216 g, $1.05 \mathrm{mmol})$ and DMAP (0.131 g, $1.07 \mathrm{mmol})$ under nitrogen atmosphere. After stirring for $2 \mathrm{~h}$ at $40{ }^{\circ} \mathrm{C}$ and $700 \mathrm{rpm}, \mathrm{P} 407$ (1.46 g, $0.10 \mathrm{mmol}$ ) was added to the solution. The reaction proceeded overnight at the same temperature, shielded from the light. Afterwards, the suspension was filtered by gravity (Prat Dumas, France) followed by dialysis (cut-off $2 \mathrm{kDa}$ ) for 7 days against PBS. Then, the solution was centrifuged (6000 rpm for $30 \mathrm{~min}$ at $25^{\circ} \mathrm{C}$, using a Beckman Allegra X 155 centrifuge from Beckman Coulter, USA). The supernatant was recovered and identified as the MTX-P407 conjugate. Quantification of MTX and P407 was performed by proton nuclear magnetic resonance $\left({ }^{1} \mathrm{H}\right.$ NMR) spectroscopy, as previously described.

\subsubsection{Synthesis of MTX-DODAB complex}

Commercial MTX was firstly converted into its carboxylic acid salt form in order to increase its water solubility. For this, MTX was suspended in water, and 2 eq. of $\mathrm{NaOH}$ were added. After complete solubilization of the solid the $\mathrm{pH}$ was neutralized. The respective MTX as sodium salt (MTX- $\mathrm{CO}_{2} \mathrm{Na}$ ) form was recovered after freeze-drying. Then, MTX- $\mathrm{CO}_{2} \mathrm{Na}(40 \mathrm{mg}, 1$ eq.) and DODAB (101 mg, 2 eq.) were dissolved in a mixture of water $(4 \mathrm{~mL})$ and ethanol $(4 \mathrm{~mL})$. The suspension was then placed in a water bath at $70{ }^{\circ} \mathrm{C}$ and $700 \mathrm{rpm}$. After $70 \mathrm{~min}$, the yellow solid formed was recovered by filtration and washed with water. This solid was identified as the MTX-DODAB complexed form with two units of DODAB, isolated with $\eta=45.5 \%$ of yield.

NMR (DMSO- $\left.d_{6}\right) \delta_{\mathrm{H}}: 0.84(\mathrm{t}, J=5.6 \mathrm{~Hz}, 12 \mathrm{H}), 1.24(\mathrm{~m}, 128 \mathrm{H}), 1.61$ (m, 9H), 1.73 (m, 3H), 3.13 (s, 3H), 3.19 (s, 12H), 3.67 (s, 1H), 4.74 (s, 2H), 6.57 (s, 2H), 6.82 (d, $J=9.2 \mathrm{~Hz}, 2 \mathrm{H}), 7.34$ (s, 1H), 7.62 (d, $J=8.8 \mathrm{~Hz}, 2 \mathrm{H}), 7.67$ (s, 1H), 8.35 (s, 1H), 8.57 (s, 1H) ppm.

\subsubsection{Synthesis of MTX-OEt}

The synthesis of MTX as di-ethylated ester form was performed using the procedure previously reported by us (Cerqueira et al., 2019).

2.2. ${ }^{1} H$ NMR

Deuterated dimethyl sulfoxide (DMSO- $d_{6}$, Cortecnet, France) was used as NMR solvent and the peak solvent used as internal reference. All spectra were traced in a $400 \mathrm{MHz}$ Bruker Avance III equipment. Pyridine ( $\geq 99.5 \%$ ) was used as internal standard for the quantification of MTX. The procedure and methodology were performed as described (Guimarães et al., 2019). Signal multiplicity is given as: s (singlet), d (doublet), $\mathrm{t}$ (triplet) and $\mathrm{m}$ (multiplet).

\subsection{MALDI-TOF}

MALDI-TOF mass spectra were acquired on a Bruker Autoflex Speed instrument (Bruker Daltonics $\mathrm{GmbH}$ ) equipped with a $337 \mathrm{~nm}$ nitrogen laser. The procedure was followed as previously described (Loureiro et al., 2018). DHB was used as matrix and the samples analysed in the linear negative mode.

\subsection{Production of P407-based nanoparticles}

The production of the nanoparticles was performed as previously described by Loureiro et al. (Loureiro et al., 2018). The initial formulations were prepared according to the purpose of the nanoparticles, where to an aqueous solution of $\mathrm{P} 407$ at $5 \mathrm{mg} / \mathrm{mL}$ in PBS (pH 7.4) was added vegetable oil (organic phase) using a 99.5/0.5 ratio (v/v), respectively. For the nanoparticles functionalized with FA at their surface, the conjugate FA-P407 was added to the aqueous phase of the initial formulation, as previously reported (Loureiro et al., 2018). Whilst for the preparation of the MTX-P407 nanoparticles, the aqueous phase was a solution of this conjugate where the concentration of the P407 was maintained at $5 \mathrm{mg} / \mathrm{mL}$. For the entrapment of the hydrophobic derivatives of MTX (MTX-DODAB complex and MTX-OEt) in the nanoparticles, these compounds were dissolved in the vegetable oil phase applied in the initial formulation. These initial formulations were submitted to homogenization cycles at high pressures (250 and 600 bar, two stages of pressure) using a high-pressure homogenizer (APV-2000 Homogenizer from SPX, Denmark), which resulted in the nanoparticles formation. The free drug was separated from the nanoparticles using PD-10 desalting columns containing Sephadex G-25 Medium (cut-off $5 \mathrm{kDa}$ ) from GE Healthcare, UK. After separation, the free drug was quantified by measuring the absorbance at $\lambda_{\max }=303 \mathrm{~nm}$. Prior to cellular tests, the nanoparticles were filtered under sterile conditions using $0.22 \mu \mathrm{m}$ polyethersulfone (PES) filter (Merck Millipore, Ireland).

\subsection{Nanoparticles characterization}

\subsubsection{Dynamic light scattering (DLS)}

The size distribution and surface charge of the produced nanoparticles were analysed by dynamic light scattering using the Malvern Zetasizer NS from Malvern Instruments, UK. The measurements were performed using PBS at pH 7.4, at $25{ }^{\circ} \mathrm{C}$ (Cerqueira et al., 2019). The concentration of P407 was kept constant at $1 \mathrm{mg} / \mathrm{mL}$. Nanoparticles were store for more than 7 months at $4{ }^{\circ} \mathrm{C}$ and constant measurements 
of size and PDI over time were performed.

\subsubsection{Nanoparticle tracking analysis (NTA)}

A quantitative analysis of the nanoparticles was assessed using a NanoSight NS500 instrument (Salisbury, UK). This system includes a charge coupled device (CCD) camera that allows visualization and tracking Brownian motion of laser-illuminated particles in suspension. The measurements were performed at room temperature and each video sequence was captured over $60 \mathrm{~s}$. Prior to analysis, the samples were 10,000 times diluted with water.

\subsection{Release profile of MTX}

The release of MTX from the nanoparticles was evaluated by dialysis (cut-off $1 \mathrm{kDa}$ ) against PBS, at two different pHs (6.5 and 7.4), in a hot room kept at $37{ }^{\circ} \mathrm{C}$. Aliquots were taken at different time points and the absorbance was measured in a 96-quartzo microplate at $303 \mathrm{~nm}$ using a Synergy Mx Multi-Mode Reader from BioTek, USA.

The release profile of the MTX from MTX-P407 nanoparticles was also assessed by the incubating the nanoparticles in a lipase from Aspergillus oryzae solution. The nanoparticles were transferred to a dialysis tube $(2 \mathrm{~mL})$ and incubated with the lipase $(50 \mu \mathrm{L}$, activity $>$ $100000 \mathrm{U} / \mathrm{g}$ ) in the same conditions described above. A control condition was performed without the addition of the lipase.

\subsection{Cellular viability assays}

\subsubsection{Cells and culture conditions}

Caco-2 cell line was maintained in a humidified atmosphere of $5 \%$ $\mathrm{CO}_{2}$ in air at $37^{\circ} \mathrm{C}$, growing in T75 flasks. Caco-2 cells were routinely grown in Dulbecco's Modified Eagle's Medium (DMEM), supplemented with $20 \%(\mathrm{v} / \mathrm{v})$ of Fetal Bovine Serum (FBS), 1\% (v/v) of penicillin/ streptomycin solution and $1 \%(\mathrm{v} / \mathrm{v})$ of Minimum Essential Medium (MEM) non-essential amino acids.

\subsubsection{Cells viability assay}

Cell viability was studied using the MTS assay, as previously described (Loureiro et al., 2015a). Briefly, Caco-2 cells were seeded in 96well tissue culture polystyrene plates at a density of $1 \times 10^{4}$ cells/well and incubated overnight to promote cell adhesion. The cells were incubated for 48 and $72 \mathrm{~h}$ with different concentrations of nanoparticles containing MTX and/or MTX derivatives and respective controls. After this incubation, an MTS mixture was added and incubated for $4 \mathrm{~h}$ at $37^{\circ} \mathrm{C}$, then the absorbance of the formazan product was read at $490 \mathrm{~nm}$. Each sample was measured in triplicate and two independent experiments were performed, being the results presented as mean value \pm standard deviation (SD). Cell viability was calculated and expressed as a percentage relative to the negative control (untreated control cells).

\subsection{Statistical analysis}

Statistical analysis was performed by one-way ANOVA with GraphPad Prism 5.0 software (La Jolla, CA, USA). Newman-Keuls Multiple Comparison test was conducted for post hoc comparisons. A P value $<0.05$ was considered to be statistically significant.

\section{Results and discussion}

\subsection{Synthesis of MTX-P407 conjugate}

The synthesis of MTX-P407 conjugate was performed through an esterification reaction. Similarly to the previous conjugate (FA-P407) synthetized by us (Loureiro et al., 2018), the reaction was carried using DCC as coupling agent, with DMAP as base source (Scheme 1).

After removal of all the unreacted MTX and the reactional components by dialysis, the expected conjugate was isolated. The concentration of P407 and MTX in the final solution was quantified by NMR (Fig. 1). NMR is a powerful tool for quantitative determination of drugs, providing high specificity and sensitivity, having great reproducibility and automation (Guimarães et al., 2019). For this quantification, pyridine was added to a certain volume of the conjugate solution (after freeze-drying) and the concentration of both components was calculated, as previously described (Guimarães et al., 2019). The results revealed a MTX concentration of $0.42 \pm 0.08 \mathrm{mg} / \mathrm{mL}$, and a P407 concentration of $29.85 \pm 0.21 \mathrm{mg} / \mathrm{mL}$. Moreover, based on the integration of the peaks corresponding to MTX and to P407 it was possible to infer $44.7 \%$ of modification of P407 with MTX. This methodology allowed, by using a single analysis, to quantify both components with high levels of reproducibility. Despite being possible to quantify MTX by absorbance analysis, no reports were found about the P407 quantification using this methodology. Thus, this technique did not seemed the ideal for the simultaneous quantification of both components on the conjugate.

In the ${ }^{1} \mathrm{H}$ NMR spectrum of MTX-P407 conjugate (Fig. 1) is possible to observe the typical signals of MTX and P407. The patterns of all signals remained the same as in the starting materials spectra. Protons near the glutamic moiety, appear at different chemical shifts, as already observed for FA-P407 conjugate (Loureiro et al., 2018).

The molecular weight of the conjugate was also assessed by MALDITOF mass spectrometry (Fig. S1, Supporting information). The MALDITOF spectra of the MTX-P407 conjugate showed a $\mathrm{m} / \mathrm{z}$ of 13372.242 Da, revealing that only one molecule of MTX was conjugated with each molecule of P407 ( $\mathrm{MW}_{\text {average }}$ of MTX-P407 conjugate - $\mathrm{MW}_{\text {average }}$ of P407 $\left.=194.467 \mathrm{Da}\right)$. The mass difference detected was lower than the MW average of MTX obtained by MALDI-TOF analysis (431.783 Da - data not shown). Considering that the spectra was acquired in negative linear mode, only average masses were detected, which might explain the differences obtained between the monomer and the conjugate (Loureiro et al., 2018).

The level of P407 modification inferred by MALDI-TOF analysis (45.0\%) was similar to the one obtained by ${ }^{1} \mathrm{H}$ NMR (44.7\%).

\subsection{Synthesis of hydrophobic MTX derivatives}

The commercial MTX presents very low solubility either in organic or aqueous solvents. Two hydrophobic MTX derivatives (MTX-DODAB complex and MTX-OEt) were produced (Scheme 2), aiming to improve its lipophilic character and increase its concentration on the organic phase (vegetable oil) of the P407-based nanoparticles.

The ionic complex between MTX and DODAB was synthetized as described in section 2.1.2. We found the addition of two equivalents of DODAB crucial for the complex formation, resulting in two units of the quaternary amine complexed with both carboxylic groups of MTX. The number of DODAB units was determined by ${ }^{1} \mathrm{H}$ NMR (Fig. 2) and ESI $(m / z=1552$ - data not shown). The ionic complex was isolated after precipitation in water $(\eta=45 \%)$. The ${ }^{1} \mathrm{H}$ NMR spectra of the ionic complex showed all the typical peaks of MTX and DODAB. The MTX peaks reveal the same pattern as previously reported (Cerqueira et al., 2019), with small chemical shifts, especially of the protons $\mathbf{g}, \mathbf{h}$ and $\mathbf{j} / \mathbf{i}$, which are close to the complexed carboxylic groups.

MTX-OEt was produced as described by Cerqueira et al. (Cerqueira et al., 2019) (Scheme 2) and its encapsulation in P407-based nanoparticles was tested without any further modification. We tested the encapsulation of this prodrug due to this hydrophobic nature but also due to its promising biological effect against cancer cells (Caco-2 cell line), previously described by Cerqueira et al. (Cerqueira et al., 2019).

\subsection{Production and characterization of the P407-based nanoparticles}

P407 features several advantages like biocompatibility and high solubility in aqueous solvents and for these reasons has been extensively applied on the production of nanodevices for diverse 


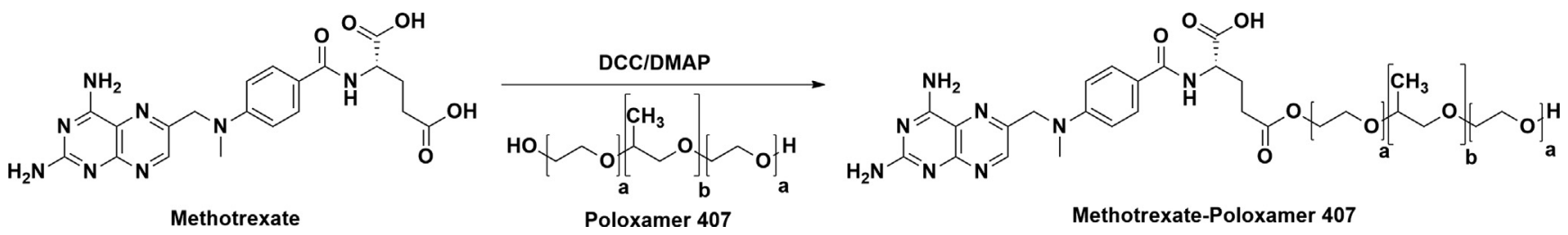

Scheme 1. Reactional scheme for the synthesis of MTX-P407 conjugate.

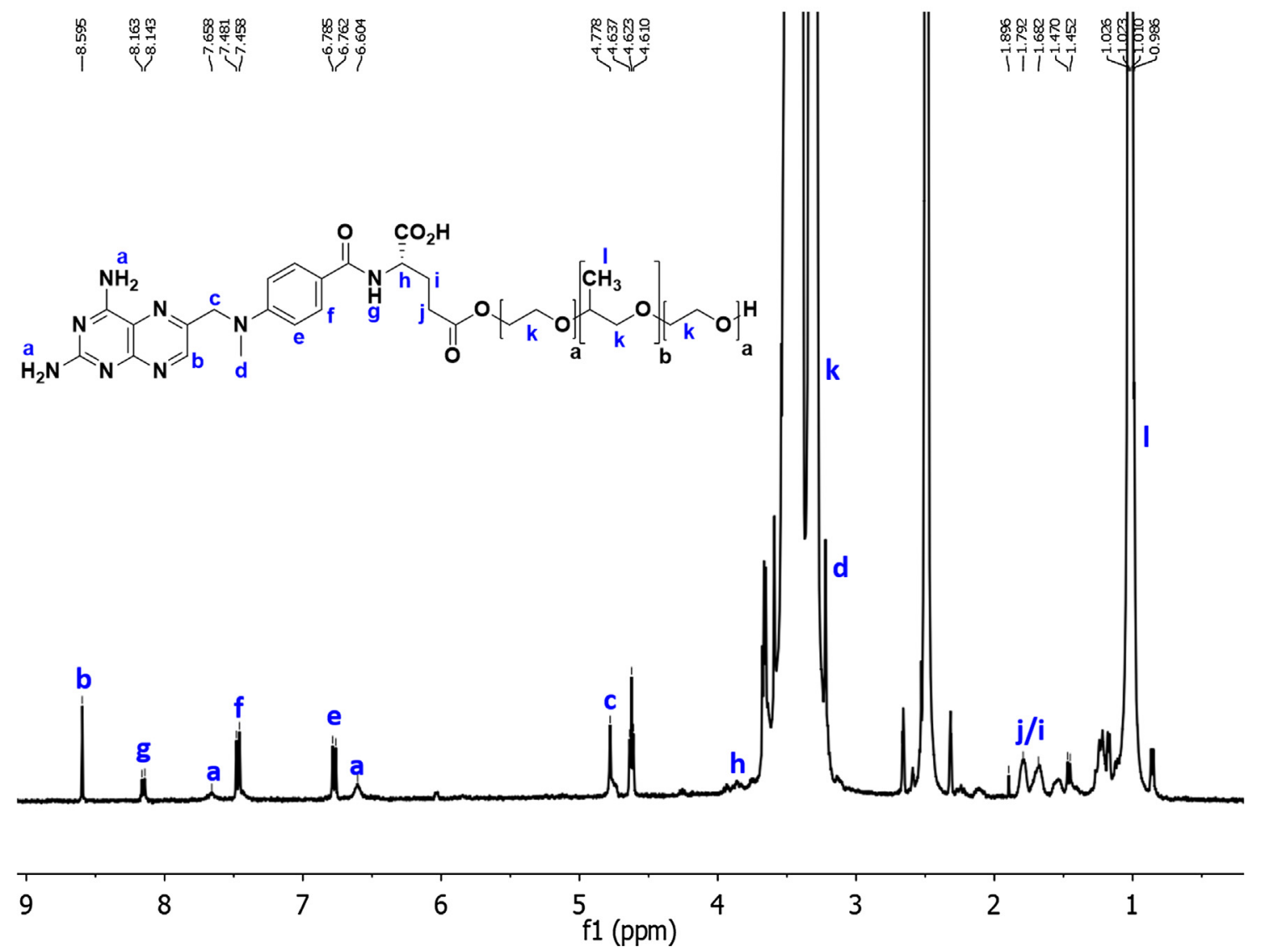

Fig. 1. ${ }^{1} \mathrm{H}$ NMR spectrum of MTX-P407 conjugate in DMSO- $d_{6}$. The peaks labelled in lowercase blue letters correspond to the protons indicated in the structures of MTX (letters from a to $\mathbf{j}$ ) and P407 (letters $\mathbf{k}$ and $\mathbf{1}$ ). (For interpretation of the references to colour in this figure legend, the reader is referred to the web version of this article.)

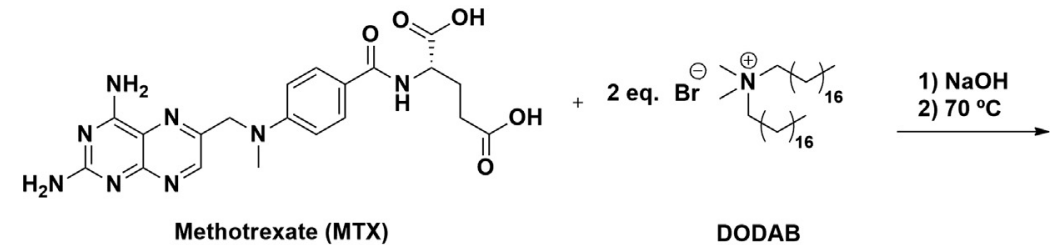

DODAB

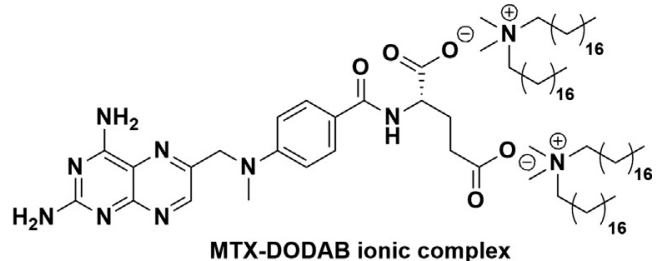

MTX-DODAB ionic complex<smiles>CCOC(=O)CCC(NC(=O)c1ccc(N(C)Cc2cnc3nc(N)nc(N)c3n2)cc1)C(=O)OCC</smiles>

Methotrexate di-ethylated (MTX-OEt)

Scheme 2. Reactional schemes for the synthesis of MTX-DODAB ionic complex and MTX-OEt. 


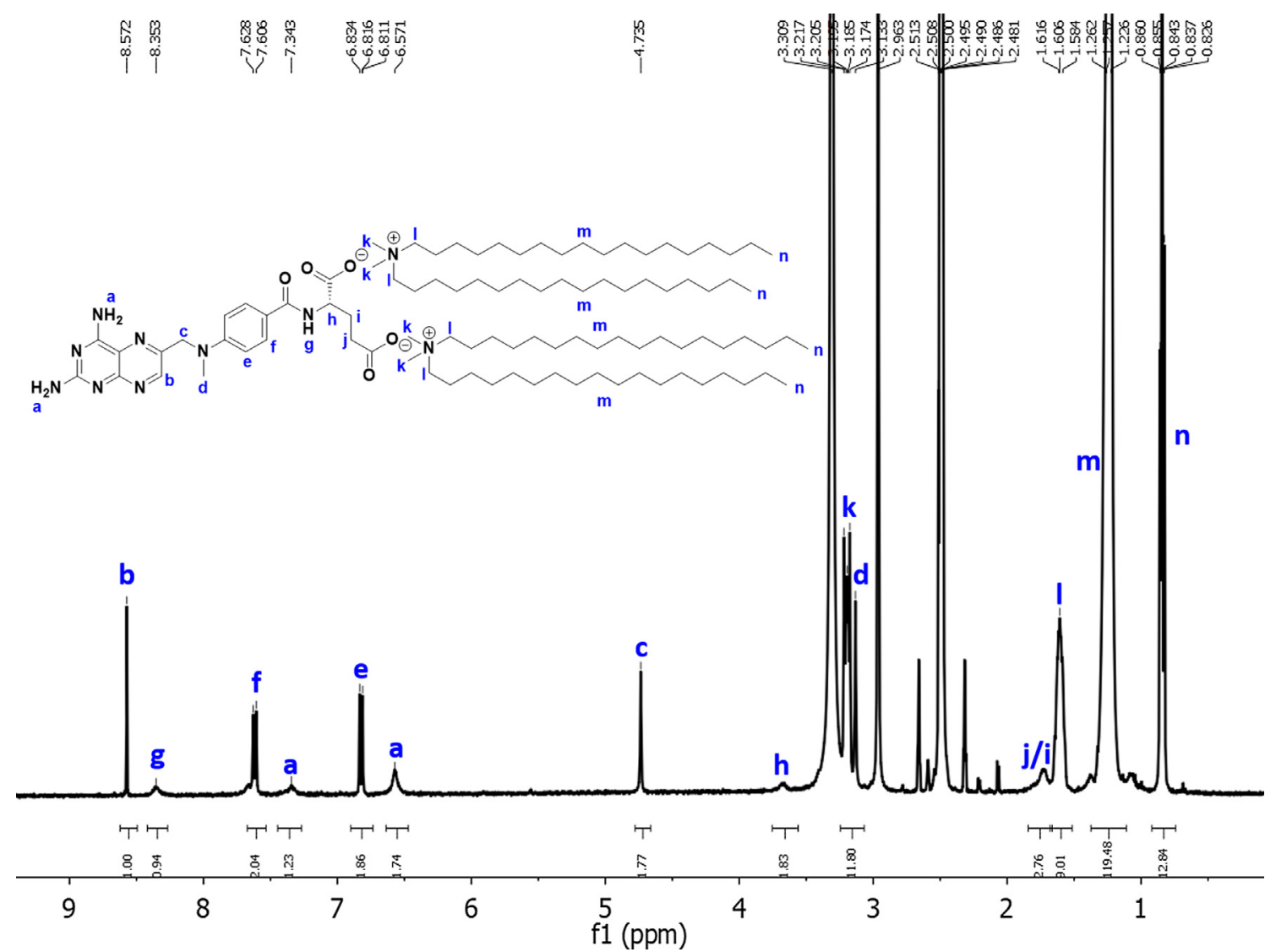

Fig. 2. ${ }^{1} \mathrm{H}$ NMR spectrum of MTX-DODAB ionic complex in DMSO- $d_{6}$. The peaks labelled in lowercase blue letters correspond to the protons indicated in the structures of MTX (letters from $\mathbf{a}$ to $\mathbf{j}$ ) and DODAB (letters from $\mathbf{k}$ to $\mathbf{n}$ ). (For interpretation of the references to colour in this figure legend, the reader is referred to the web version of this article.)

applications (Breitenbach et al., 2017). Loureiro et al. described the use of the high pressure homogenization method to produce P407-based nanoparticles with suitable properties for therapeutic applications (Loureiro et al., 2018). These nanoparticles showed potentiality for intravenous (IV) administration (small and stealth nanoparticles) and demonstrated the ability to be efficiently internalized by cancer cells.

Nanoparticles larger than $100 \mathrm{~nm}$ can be easily cleared-off by macrophages and other mononuclear phagocyte system (MPS) cells and cleared from circulation by phagocytosis (Zahr et al., 2006). Additionally, nanoparticles below $10 \mathrm{~nm}$ have a higher tendency to be excreted by the kidneys (Danaei et al., 2018). Therefore, the range between $10 \mathrm{~nm}$ and $100 \mathrm{~nm}$ is considered the most appropriate for IV administration to reach the target cells (Loureiro et al., 2015b). Populations presenting polydispersity values $(\mathrm{PDI}) \leq 0.2$ are considered acceptable for polymer-based nanoparticles (Danaei et al., 2018), representing monodisperse populations of nanoparticles (Cun et al., 2010; Hornig et al., 2009) with the ideal physicochemical conditions for IV administration.

In this work, empty P407 based-nanoparticles functionalized or nonfunctionalized with FA were produced and their performance was compared with the nanoparticles produced using the MTX-P407 conjugate. The physicochemical characterization of the nanoparticles performed by DLS and NTA techniques (Table 1) indicate that these nanoparticles present suitable characteristics for IV administration. All formulations revealed similar small size (91-98 nm) and monodisperse populations (PDI $<0.2$ ), indicating that the MTX-P407 conjugate did not influence negatively the nanoparticles formation, leading, on the other hand, to the formation of higher concentration of particles (Table 1, NTA results).

\subsection{Encapsulation of MTX-derivatives in P407-based nanoparticles}

The hydrophobic derivatives of MTX (MTX-DODAB and MTX-OEt), as well as the unmodified MTX, were encapsulated in the produced nanoparticles (Table 2). Due to its poor solubility in organic or aqueous media, the lowest final drug concentration was obtained when encapsulating unmodified MTX. As expected, the highest amount of MTX/ MTX derivative on the final formulation was achieved for the MTXP407 nanoparticles loaded with MTX-OEt. The amount of MTX on the loaded formulations derives from MTX linked to P407 at the nanoparticles' shell, and from MTX-OEt entrapped onto the nanoparticles. For this reason, the molar concentration of MTX/MTX derivative was not inferred since the formulation is composed by two different types of MTX which cannot be distinguished using the indirect absorbance quantification method applied herein.

From Table 2 one can also highlight that all the loaded nanoparticles reveal sizes similar to the empty nanoparticles (Table 1) corresponding to monodisperse populations $(<0.150)$. All formulations have neutral surface charge (zeta-potential values around $0 \pm 2 \mathrm{mV}$ ). The neutral and hydrophilic surface, conferred by the presence of PEG chains of the P407, can lead to a longer half-life in the blood circulation (Zahr et al., 2006), which in turns the developed formulations suitable for IV therapeutic applications. The formulations revealed also high stability along time, demonstrated by the maintenance of their physicochemical properties even after 7 months of storage (data not shown).

Considering the highest concentration of drug at the final formulations, the P407 nanoparticles encapsulating MTX-OEt (functionalized with FA for a specific delivery) or the MTX-P407 nanoparticles encapsulating MTX-OEt were chosen for posterior assays. 
Table 1

Physicochemical characterization of P407-based nanoparticles functionalized and nonfunctionalized with FA and of MTX-P407 nanoparticles, measured by DLS and NTA. Values represent the mean \pm SD of two independent experiments.

\begin{tabular}{|c|c|c|c|c|c|}
\hline & \multicolumn{2}{|l|}{ DLS } & \multicolumn{3}{|l|}{ NTA } \\
\hline & Z-average (nm) & Polydispersity index (PDI) & Mean (nm) & $\mathrm{SD}(\mathrm{nm})$ & Conc, particles (particles/mL) \\
\hline P407 nanoparticles & $98.4 \pm 2.9$ & $0.176 \pm 0.041$ & $98.0 \pm 10.1$ & $43.5 \pm 16.8$ & $2.8 \pm 0.38\left(\mathrm{E}^{12}\right)$ \\
\hline FA-tagged P407 nanoparticles & $92.3 \pm 3.7$ & $0.118 \pm 0.001$ & $98.8 \pm 8.4$ & $43.5 \pm 15.1$ & $2.7 \pm 0.23\left(\mathrm{E}^{12}\right)$ \\
\hline MTX-P407 nanoparticles & $91.3 \pm 24.4$ & $0.122 \pm 0.003$ & $97.0 \pm 5.7$ & $39.0 \pm 3.9$ & $5.1 \pm 0.61\left(\mathrm{E}^{12}\right)$ \\
\hline
\end{tabular}

Table 2

Physicochemical characterization of the nanoparticles (evaluated by DLS) and final drug concentration in the formulations. Values represent the mean \pm SD of two independent experiments.

\begin{tabular}{|c|c|c|c|c|c|}
\hline \multirow[t]{2}{*}{ P407-based nanoparticles } & \multirow[t]{2}{*}{ MTX encapsulated } & \multirow[t]{2}{*}{ Z-average $(\mathrm{d} \cdot \mathrm{nm})$} & \multirow[t]{2}{*}{ PDI } & \multicolumn{2}{|c|}{ [MTX/MTX derivatives] at the final formulations* } \\
\hline & & & & $\mathrm{mg} / \mathrm{mL}$ & $\mathrm{mM}^{\mathrm{k*k}}$ \\
\hline P407 & Unmodified MTX & $97.3 \pm 10.8$ & $0.105 \pm 0.020$ & $0.009 \pm 0.004$ & $0.020 \pm 0.008$ \\
\hline P407 & MTX-DODAB & $94.7 \pm 4.1$ & $0.094 \pm 0.013$ & $0.066 \pm 0.001$ & $0.042 \pm 0.000$ \\
\hline FA-tagged P407 & MTX-DODAB & $95.1 \pm 7.9$ & $0.099 \pm 0.008$ & $0.067 \pm 0.001$ & $0.043 \pm 0.000$ \\
\hline P407 & MTX-OEt & $86.7 \pm 6.7$ & $0.096 \pm 0.013$ & $0.041 \pm 0.018$ & $0.078 \pm 0.040$ \\
\hline FA- tagged P407 & MTX-OEt & $86.1 \pm 1.7$ & $0.096 \pm 0.023$ & $0.032 \pm 0.006$ & $0.059 \pm 0.017$ \\
\hline MTX-P407 & - & $91.3 \pm 24.4$ & $0.122 \pm 0.003$ & $0.031 \pm 0.005$ & $0.067 \pm 0.011$ \\
\hline MTX-P407 & MTX-OEt & $84.0 \pm 12.7$ & $0.132 \pm 0.003$ & $0.046 \pm 0.003$ & $-e^{* k * k * k}$ \\
\hline
\end{tabular}

* Concentration of drug in the nanoparticles after dilution of 1.4x in the PD-10 desalting columns.

** The molar concentration was also calculated because the MTX-DODAB complex presents a very high molecular weight (1554.52 $\mathrm{g} / \mathrm{mol})$ comparatively to the MTX $(454.44 \mathrm{~g} / \mathrm{mol})$ and the MTX-OEt $(510.55 \mathrm{~g} / \mathrm{mol})$, resulting in a great difference on the molar concentration.

$* * *$ Not possible to determine.

\subsection{Release profile of MTX from P407-based nanoparticles}

The in vitro release of MTX from the P407-based nanoparticles loaded with MTX-OEt was evaluated at $37{ }^{\circ} \mathrm{C}$ through dialysis of the MTX from the bulk formulations and after incubation of the MTX-P407 nanoparticles with a lipase (Fig. 3). The release of MTX was performed at two different $\mathrm{pHs}$, at physiological $\mathrm{pH}$ (7.4, full line) and at acidic $\mathrm{pH}$, mimicking the tumor microenvironment (6.5, dash line) (Cao et al., 2017; Lei et al., 2016; Zhang et al., 2016).

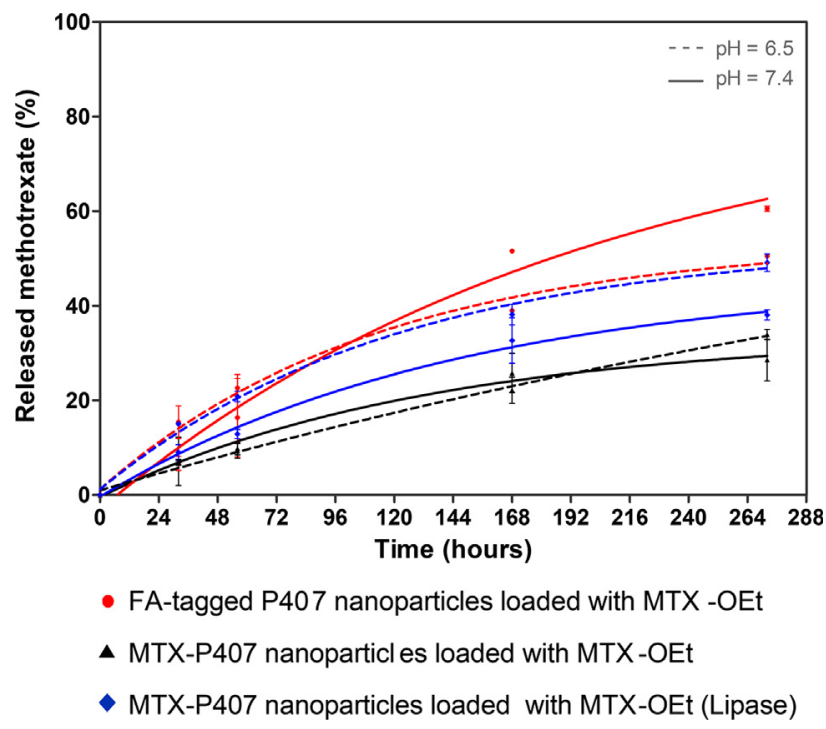

Fig. 3. Release over time of MTX from the P407 nanoparticles functionalized with FA and MTX-P407 nanoparticles, both loaded with MTX-OEt (red and black lines, respectively), and release of MTX from the MTX-P407 nanoparticles in the presence of lipase from Aspergillus oryzae (blue lines). Physiological $\mathrm{pH}$ (7.4, full line) and acidic pH (6.5, dash line). The values are a mean of two independent experiments. (For interpretation of the references to colour in this figure legend, the reader is referred to the web version of this article.)
Many nanodevices have been studied for the encapsulation and release of MTX, however most of the strategies revealed the release of all the drug content after 48 h (Karasulu et al., 2007; Nogueira et al., 2016; Zhao et al., 2016).

The P407-based nanoparticles tested, as functionalized with FA or prepared with MTX-P407 conjugate, revealed a similar slow release profile, at both $\mathrm{pH}$ tested, as depicted in Fig. 3. As P407 is a non-ionic polymer it would be expected that small differences in the buffer $\mathrm{pH}$ would not affect the stability of the nanoparticles, and therefore their release profile.

FA-tagged P407 nanoparticles showed the highest MTX release at both $\mathrm{pH}$ tested ( $>50 \%$ after $272 \mathrm{~h}$, Fig. 3, red lines) than the nanoparticles prepared using the MTX-P407 conjugate $(\approx 30 \%$ after $272 \mathrm{~h}$, Fig. 3, black lines). The data is presented in terms of MTX percentage release relatively to the total amount in the final formulations. Considering that MTX-P407 nanoparticles are composed by a higher amount MTX/MTX derivative (entrapped MTX-OEt and MTX conjugated to P407), when comparing with the FA-tagged P407 nanoparticles, a lower percentage of release after $272 \mathrm{~h}$ is observed. We might speculate that the MTX-OEt present in the hydrophobic core of both formulations is more easily released from the nanoparticles than the MTX covalently linked to P407, which would be more hardly disrupted. In this way, the FA-tagged nanoparticles release a higher percentage of MTX, than the formulations composed by the MTX-P407 conjugate.

In a biological environment, the nanoparticles are susceptible to degradation by a panoply of hydrolytic enzymes (Rios-Ramírez, 2013), being esterases an example of enzymes involved in drug metabolism (Fukami and Yokoi, 2012). In this way, the role of lipase from Aspergillus oryzae on the MTX-P407 conjugate hydrolysis was undertaken (Fig. 3, blue lines). We chose this lipase based on its affinity for large substrates, such as acylglycerols (Toida et al., 1995). During all the dialysis processing, the enzyme remained stable (no significant activity loss until 1 month at $37^{\circ} \mathrm{C}$ ). At pH 7.4, MTX-P407 nanoparticles loaded with MTX-OEt, even after incubation with lipase, revealed a lower MTX release $(\approx 38 \%$ after $272 \mathrm{~h}$ ) than observed for the FA-tagged P407 nanoparticles. At $\mathrm{pH}$ 6.5, the enzyme action was responsible for an 
increase of the MTX release from the MTX-P407 nanoparticles loaded with MTX-OEt to the levels obtained for the FA-tagged P407 nanoparticles ( $\approx 50 \%$ after $272 \mathrm{~h}$ of incubation).

The presence of lipase incremented only $10 \%(\mathrm{pH}=7.4)$ and $15 \%$ $(\mathrm{pH}=6.5)$ of MTX release from MTX-P407 nanoparticles. Considering the low release increment promoted by lipase, which in theory would break the linkage between P407 and MTX, one might speculate that the MTX-P407 formulations are composed mainly by MTX-OEt. This MTX derivative, at the time of particles formation, is dissolved in vegetable oil being entrapped onto the hydrophobic core, which in turns its release slow, as in absence or in presence of enzyme.

Both nanoparticles (FA-tagged P407 and MTX-P407 nanoparticles) revealed a controlled and sustained drug release demonstrating potentiality to serve as release matrixes, reducing the dose frequency and increasing the patient compliance.

\subsection{Biological effect of P407-based nanoparticles loaded with MTX-OEt}

Previous studies revealed that P407-based nanoparticles (up to $900 \mu \mathrm{g} / \mathrm{mL}$ ) did not induce cytotoxicity in immortalized human normal cells, even after $72 \mathrm{~h}$ of incubation (Loureiro et al., 2018). For this reason, we can consider that these nanoparticles are not harmful to normal cells, being promising candidates for therapeutic applications. In order to evaluate the biological effect of the P407-based nanoparticles loaded with MTX-OEt (functionalized or not with FA), the cell viability of cancer cells was assessed. Caco- 2 cell line was chosen for the biological evaluation since it endogenously express folate receptor (FR) (Doucette and Stevens, 2001).

The results demonstrated that, as expected, empty nanoparticles (with or without FA) did not induce a significant loss of cell viability (Fig. 4). The free prodrug (MTX-OEt) or loaded into the nanoparticles displayed very similar effect on the cell viability $(\approx 70 \%)$, confirming the ability of nanoparticles to release the MTX derivative when internalized by the cells. The FA-tagged nanoparticles loaded with $5 \mu \mathrm{g} / \mathrm{mL}$ of MTX-OEt showed a more pronounced biological effect against cancer

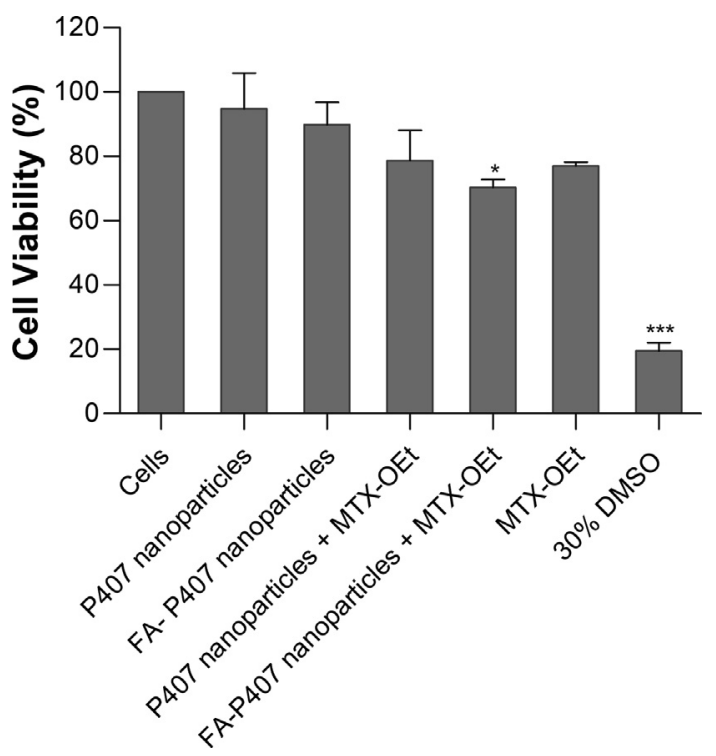

Fig. 4. Caco-2 cell viability after 48 h of contact with P407-based nanoparticles (functionalized or nonfunctionalized with FA) containing or not $5 \mu \mathrm{g} / \mathrm{mL}$ of MTX-OEt and with the MTX derivative alone at the same concentration, compared with cells (negative control) and cells incubated with $30 \%(\mathrm{v} / \mathrm{v})$ of DMSO (death control), determined by MTS assay. Values are the mean \pm SD of two independent experiments. Statistical significant differences from the negative control are indicated as: * P-value $<0.05$ and $* * *$ P-value $<0.0001$. Values of FA-P407 nanoparticles + MTX-OEt and MTX-OEt alone are not statistically significant.

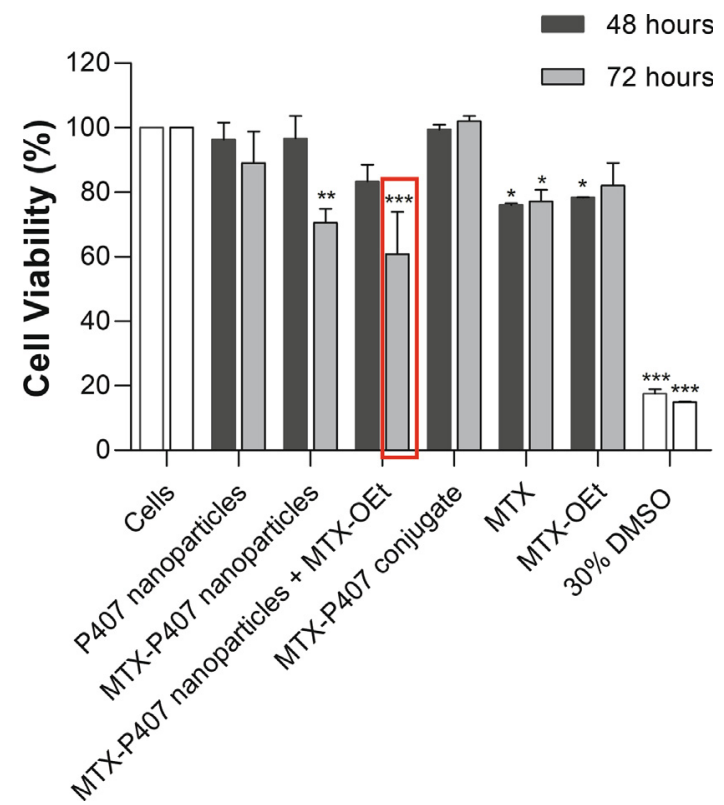

Fig. 5. Caco-2 cell viability after 48 and $72 \mathrm{~h}$ of contact with: empty MTX-P407 nanoparticles; MTX-P407 nanoparticles loaded with MTX-OEt; MTX-P407 conjugate; and the two types of MTX (unmodified MTX and MTX-OEt), at the same concentration ( $8 \mu \mathrm{g} / \mathrm{mL}$ ), compared with cells (negative control) and cells incubated with $30 \%(\mathrm{v} / \mathrm{v})$ of DMSO (death control), determined by MTS assay. Values are the mean \pm SD of two independent experiments. Statistical significant differences from the negative control are indicated as: ${ }^{*}$ P-value $<$ $0.05 ; * *$ P-value $<0.01$ and $* * *$ P-value $<0.0001$

cells, comparing with the effect of the same concentration of free MTXOEt (but not statistically significant). We might deduce that these FAtagged nanoparticles were recognized by the FR at the surface of Caco-2 cells being internalized by FR-mediated endocytosis, releasing further the drug as intended.

The biological effect of the P407-based nanoparticles loaded with MTX-OEt was not significant due to the low concentration of drug applied. In order to increase the total concentration of drug applied (to $8 \mu \mathrm{g} / \mathrm{mL}$ ) in Caco-2 cells, the biological effect of the MTX-P407 nanoparticles was also evaluated. Fig. 5 shows the Caco- 2 cell viability after 48 and $72 \mathrm{~h}$ of incubation with: empty MTX-P407 nanoparticles, MTXP407 nanoparticles loaded with MTX-OEt and respective controls.

The results revealed that all the tested nanoparticles and the MTXP407 conjugate did not induce a significant loss of cell viability after $48 \mathrm{~h}$ of incubation. However, after $72 \mathrm{~h}$ of incubation, the MTX-P407 nanoparticles loaded with MTX-OEt induced approximately $40 \%$ of cell death, indicating disruption or release of MTX/MTX derivative from the nanoparticles. The biological effect observed only after $72 \mathrm{~h}$ of incubation, can be explained by the slow release profile of MTX during the dialysis studies. The free MTX and MTX-OEt demonstrated lower cytotoxicity than when entrapped onto the nanoparticles, confirming the role of the nanodevices on the internalization and release of the drug inside the cells. The cells incubated with the MTX-P407 conjugate showed $100 \%$ of cell viability even after $72 \mathrm{~h}$ of incubation. One might claim that the produced conjugate, in the free form, is not internalized by the cells and consequently is not subjected to the action the cell machinery. Since no internalization and disruption occurred, this conjugate did not induce biological effect on cancer cells.

The slow MTX release profile observed previously, together with the great biological effect against cancer cells, revealed MTX-P407 nanoparticles loaded with the hydrophobic MTX derivative (MTX-OEt) as a new outstanding methodology for cancer therapy. 


\section{Conclusions}

P407-based nanoparticles loaded with different MTX derivatives were successfully produced. These nanoparticles remained stable for more than 7 months of storage, maintaining their physicochemical proprieties suitable for IV therapeutic applications. The encapsulation of two hydrophobic MTX derivatives (MTX-OEt and MTX-DODAB) into the hydrophobic core of the nanoparticles revealed MTX-OEt as the best prodrug to increase the final drug cargo on the developed formulations. The P407-based nanoparticles loaded with MTX-OEt revealed a slow drug release profile, which confers a great advantage relatively to many nanodevices recently studied for the encapsulation and release of MTX. MTX-P407 nanoparticles loaded with MTX-OEt showed a great biological effect against cancer cells, demonstrating to be more effective than the same concentration of free MTX. All these findings allow us to envisage the IV therapeutic application of these nanoparticles in order to potentiate the MTX therapeutic effect and overcome its several limitations. Thus, the developed nanoparticles demonstrated several potentialities to be implemented as a drug delivery system in cancer therapy.

\section{CRediT authorship contribution statement}

Sofia Moura: Investigation, Formal analysis, Writing - original draft. Jennifer Noro: Investigation, Formal analysis, Writing - original draft. Patrícia Cerqueira: Investigation. Carla Silva: Conceptualization, Writing - review \& editing. Artur Cavaco-Paulo: Conceptualization, Resources, Supervision, Funding acquisition. Ana Loureiro: Conceptualization, Methodology, Validation, Writing - review \& editing, Supervision, Project administration.

\section{Declaration of Competing Interest}

The authors declare that they have no known competing financial interests or personal relationships that could have appeared to influence the work reported in this paper.

\section{Acknowledgements}

This study was supported by the Portuguese Foundation for Science and Technology (FCT) under the scope of the strategic funding of UID/ BIO/04469/2019 unit and BioTecNorte operation (NORTE-01-0145FEDER-000004) funded by the European Regional Development Fund under the scope of Norte2020 - Programa Operacional Regional do Norte. The authors also thanks to FCT for funding their scholarship: Jennifer Noro (SFRH/BD/121673/2016) and Carla Silva (SFRH/IF/ 00186/2015). This work has also received funding from the European Union Horizon 2020 research and innovation program under grant agreement NMP-06-2015-683356 FOLSMART.

\section{Appendix A. Supplementary material}

Supplementary data to this article can be found online at https:// doi.org/10.1016/j.ijpharm.2019.118924.

\section{References}

Abolmaali, S.S., Tamaddon, A., Yousefi, G., Javidnia, K., Dinarvand, R., 2014. Sequential optimization of methotrexate encapsulation in micellar nano-networks of polyethyleneimine ionomer containing redox-sensitive cross-links. Int. J. Nanomed. 9, 2833-2848.

Alam, F., Naim, M., Aziz, M., Yadav, N., 2015. Unique roles of nanotechnology in medicine and cancer-II. Indian J. Cancer 52, 1-9.

Alexis, F., Pridgen, E., Molnar, L.K., Farokhzad, O.C., 2008. Factors Affecting the Clearance and Biodistribution of Polymeric Nanoparticles. Mol. Pharm. 5, 505-515.

Bodratti, A.M., Alexandridis, P., 2018. Formulation of Poloxamers for Drug Delivery. J. Funct. Biomater. 9, 11-35.

Breitenbach, B.B., Schmid, I., Wich, P.R., 2017. Amphiphilic polysaccharide block copolymers for $\mathrm{pH}$-responsive micellar nanoparticles. Biomacromolecules 18, 2839-2848.

Cao, Y., Liu, F., Chen, Y., Yu, T., Lou, D., Guo, Y., Li, P., Wang, Z., Ran, H., 2017. Drug release from core-shell PVA/silk fibroin nanoparticles fabricated by one-step electrospraying. Sci. Rep. 7, 11913.

Cerqueira, P., Noro, J., Moura, S., Guimarães, D., Silva, C., Cavaco-Paulo, A., Loureiro, A. 2019. PTS micelles for the delivery of hydrophobic methotrexate. Int. J. Pharm.

Chen, Y., Sha, X., Zhang, W., Zhong, W., Fan, Z., Ren, Q., Chen, L., Fang, X., 2013. Pluronic mixed micelles overcoming methotrexate multidrug resistance: in vitro and in vivo evaluation. Int. J. Nanomed. 8, 1463-1476.

Choi, G., Kim, T.-H., Oh, J.-M., Choy, J.-H., 2018. Emerging nanomaterials with advanced drug delivery functions; focused on methotrexate delivery. Coord. Chem. Rev. 359, $32-51$.

Cun, D., Foged, C., Yang, M., Frøkjær, S., Nielsen, H.M., 2010. Preparation and characterization of poly(dl-lactide-co-glycolide) nanoparticles for siRNA delivery. Int. J. Pharm. 390, 70-75.

Danaei, M., Dehghankhold, M., Ataei, S., Hasanzadeh Davarani, F., Javanmard, R., Dokhani, A., Khorasani, S., Mozafari, M.R., 2018. Impact of particle size and polydispersity index on the clinical applications of lipidic nanocarrier systems. Pharmaceutics 10, 57-74.

Doucette, M.M., Stevens, V.L., 2001. Folate receptor function is regulated in response to different cellular growth rates in cultured mammalian cells. J. Nutrition 131, 2819-2825.

Fukami, T., Yokoi, T., 2012. The emerging role of human esterases. Drug Metab. Pharmacokinet. 27, 466-477.

Guimarães, D., Noro, J., Loureiro, A., Cavaco-Paulo, A., Nogueira, E., 2019. Quantification of drugs encapsulated in liposomes by 1H NMR. Colloids Surf., B 179, 414-420.

Gulfam, M., Matini, T., Monteiro, P.F., Riva, R., Collins, H., Spriggs, K., Howdle, S.M., Jérôme, C., Alexander, C., 2017. Bioreducible cross-linked core polymer micelles enhance in vitro activity of methotrexate in breast cancer cells. Biomater. Sci. 5, 532-550.

Hornig, S., Heinze, T., Becer, C.R., Schubert, U.S., 2009. Synthetic polymeric nanoparticles by nanoprecipitation. J. Mater. Chem. 19, 3838-3840.

Huang, D., Zhuang, Y., Shen, H., Yang, F., Wang, X., Wu, D., 2018. Acetal-linked PEGylated paclitaxel prodrugs forming free-paclitaxel-loaded pH-responsive micelles with high drug loading capacity and improved drug delivery. Mater. Sci. Eng., C 82, $60-68$.

Kabanov, A.V., Batrakova, E.V., Alakhov, V.Y., 2002a. Pluronic ${ }^{\circledR}$ block copolymers as novel polymer therapeutics for drug and gene delivery. J. Control. Release 82, 189-212.

Kabanov, A.V., Batrakova, E.V., Alakhov, V.Y., 2002b. Pluronic ${ }^{\circledR}$ block copolymers for overcoming drug resistance in cancer. Adv. Drug Deliv. Rev. 54, 759-779.

Karasulu, H.Y., Karabulut, B., Göker, E., Güneri, T., Gabor, F., 2007. Controlled release of methotrexate from W/O microemulsion and its in vitro antitumor activity. Drug Delivery 14, 225-233.

Kedar, U., Phutane, P., Shidhaye, S., Kadam, V., 2010. Advances in polymeric micelles for drug delivery and tumor targeting. Nanomed.: Nanotechnol. Biol. Med. 6, 714-729.

Lei, Y., Hamada, Y., Li, J., Cong, L., Wang, N., Li, Y., Zheng, W., Jiang, X., 2016. Targeted tumor delivery and controlled release of neuronal drugs with ferritin nanoparticles to regulate pancreatic cancer progression. J. Control. Release 232, 131-142.

Loureiro, A., Abreu, A.S., Sárria, M.P., Figueiredo, M.C.O., Saraiva, L.M., Bernardes, G.J.L., Gomes, A.C., Cavaco-Paulo, A., 2015a. Functionalized protein nanoemulsions by incorporation of chemically modified BSA. RSC Adv. 5, 4976-4983.

Loureiro, A., Azoia, N.G., Gomes, A.C., Cavaco-Paulo, A., 2016. Albumin-based nanodevices as drug carriers. Curr. Pharm. Des. 22, 1371-1390.

Loureiro, A., Nogueira, E., Azoia, N.G., Sárria, M.P., Abreu, A.S., Shimanovich, U., Rollett, A., Härmark, J., Hebert, H., Guebitz, G., Bernardes, G.J.L., Preto, A., Gomes, A.C., Cavaco-Paulo, A., 2015b. Size controlled protein nanoemulsions for active targeting of folate receptor positive cells. Colloids Surf., B 135, 90-98.

Loureiro, A., Noro, J., Abreu, A.S., Nogueira, E., Soares da Costa, D., Silva, C., CavacoPaulo, A., 2018. Absence of albumin improves in vitro cellular uptake and disruption of poloxamer 407-based nanoparticles inside cancer cells. Mol. Pharm. 15, 527-535.

Moura, J.A., Valduga, C.J., Tavares, E.R., Kretzer, I.F., Maria, D.A., Maranhão, R.C., 2011 Novel formulation of a methotrexate derivative with a lipid nanoemulsion. Int. J. Nanomed. 6, 2285-2295.

Nogueira, D.R., Scheeren, L.E., Macedo, L.B., Marcolino, A.I.P., Pilar Vinardell, M., Mitjans, M., Rosa Infante, M., Farooqi, A.A., Rolim, C.M.B., 2016. Inclusion of a pHresponsive amino acid-based amphiphile in methotrexate-loaded chitosan nanoparticles as a delivery strategy in cancer therapy. Amino Acids 48, 157-168.

Pinto Reis, C., Neufeld, R.J., Ribeiro, A.J., Veiga, F., 2006. Nanoencapsulation I. Methods for preparation of drug-loaded polymeric nanoparticles. Nanomed.: Nanotechnol. Biol. Med. 2, 8-21.

Ren, J., Fang, Z., Yao, L., Dahmani, F.Z., Yin, L., Zhou, J., Yao, J., 2015. A micelle-like structure of poloxamer-methotrexate conjugates as nanocarrier for methotrexate delivery. Int. J. Pharm. 487, 177-186.

Rios-Ramírez, E.T.A.A., 2013. Biodegradation of medical purpose polymeric materials and their impact on biocompatibility. Biodegradation-Life Sci. 1-29.

Rizvi, S.A.A., Saleh, A.M., 2018. Applications of nanoparticle systems in drug delivery technology. Saudi Pharm. J. 26, 64-70.

Toida, J., Kondoh, K., Fukuzawa, M., Ohnishi, K., Sekiguchi, J., 1995. Purification and Characterization of a Lipase from Aspergillus oryzae. Biosci. Biotechnol. Biochem. 59, 1199-1203.

Xie, J., Fan, Z., Li, Y., Zhang, Y., Yu, F., Su, G., Xie, L., Hou, Z., 2018. Design of pHsensitive methotrexate prodrug-targeted curcumin nanoparticles for efficient dualdrug delivery and combination cancer therapy. Int. J. Nanomed. 13, 1381-1398. 
Zahr, A.S., Davis, C.A., Pishko, M.V., 2006. Macrophage uptake of core-shell nanoparticles surface modified with poly(ethylene glycol). Langmuir 22, 8178-8185.

Zhang, M., Xu, C., Wen, L., Han, M.K., Xiao, B., Zhou, J., Zhang, Y., Zhang, Z., Viennois, E., Merlin, D., 2016. A hyaluronidase-responsive nanoparticle-based drug delivery system for targeting colon cancer cells. Cancer Res. 76, 7208-7218.
Zhao, Y., Guo, Y., Li, R., Wang, T., Han, M., Zhu, C., Wang, X., 2016. Methotrexate nanoparticles prepared with codendrimer from polyamidoamine (PAMAM) and oligoethylene glycols (OEG) dendrons: antitumor efficacy in vitro and in vivo. Sci. Rep. 6, 28983. 